\title{
Velocity Fields of a Filament Region Observed with Ground-Based Telescopes and from SOHO
}

P. Mein, B. Schmieder ${ }^{1}$ and J.-M. Malherbe

Observatoire de Paris, Section de Meudon, 5 Place Janssen, F-92195 Meudon, France

J.E. Wiik, O. Engvold, P. Brekke

Inst. Theoret. Astrophys., University of Oslo, P.O. Box 1029, Blindern, N-0315 Oslo, Norway

J.B. Zirker

NSO, NOAO, Sunspot, New Mexico 88349, USA

A.I. Poland

NASA/Goddard Space Flight Center, Greenbelt, Maryland, USA

J.-P. Delaboudinière

Inst. d'Astrophysique Spatiale, Université Paris XI, F-91405 Orsay Cedex, France

J. Staiger

K.I.S., Schöneckstrasse 6, D-79104 Freiburg, Germany

Abstract. We present preliminary results on solar filaments observed from the ground and from SOHO in September 1996. Motions in the network and close to the filament "barbs" are shown at different levels of the chromosphere and the transition region.

During the International Campaign of September 1996 several large filaments were observed simultaneously from the ground (MSDP of the German VTT, Tenerife, and Swedish Vacuum Solar Telescope [SVST], La Palma) and from SOHO (JOPs 17 and 29, SUMER, CDS and EIT). The observations on September 25, 1996 covered a large filament with well defined barbs (feet). We present a few combined results from these instruments. The MSDP produced $\mathrm{H}_{\alpha}$ and CaII $8542 \AA$ line profiles covering two strips of $170 \times 600 \mathrm{arc} \mathrm{sec}^{2}$. High resolution $\mathrm{H}_{\alpha}$ filtergrams were obtained with the SVST. Spectral scans with SUMER and CDS on SOHO produced dopplergrams in the temperature range from $3 \times 10^{4} \mathrm{~K}$ to several $10^{5} \mathrm{~K}$. The data show the connections between smallscale velocities and thread structures of the filament "barbs".

\footnotetext{
${ }^{1}$ Inst. Theoret. Astrophys., University of Oslo, P.O. Box 1029, Blindern, N-0315 Oslo, Norway
} 


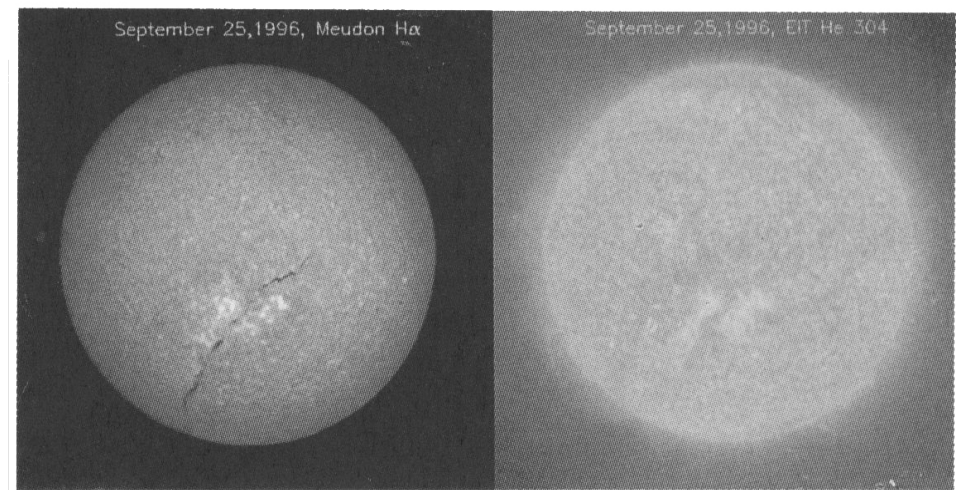

Figure 1. $\mathrm{H}_{\alpha}$ Meudon spectroheliogram and EIT He $304 \AA$ image, showing the full filament channel across the southern hemisphere. The following figures ( 2 to 4 ) concern only the northern part of the filament, close to the disk center.

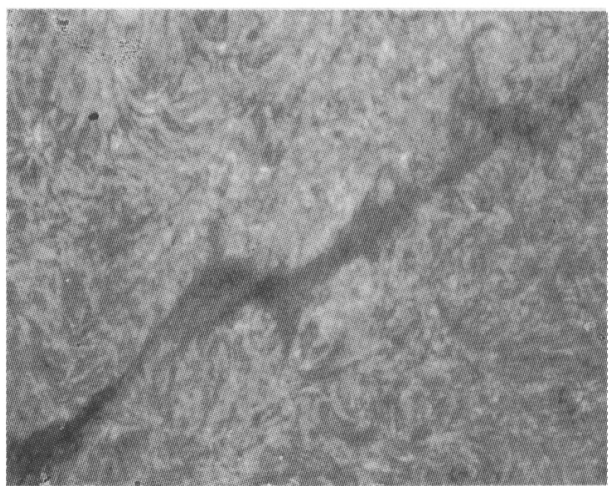

Figure 2. High resolution $\mathrm{H}_{\alpha}$ image of the filament at 12:03:25 UT (SVST, La Palma). 

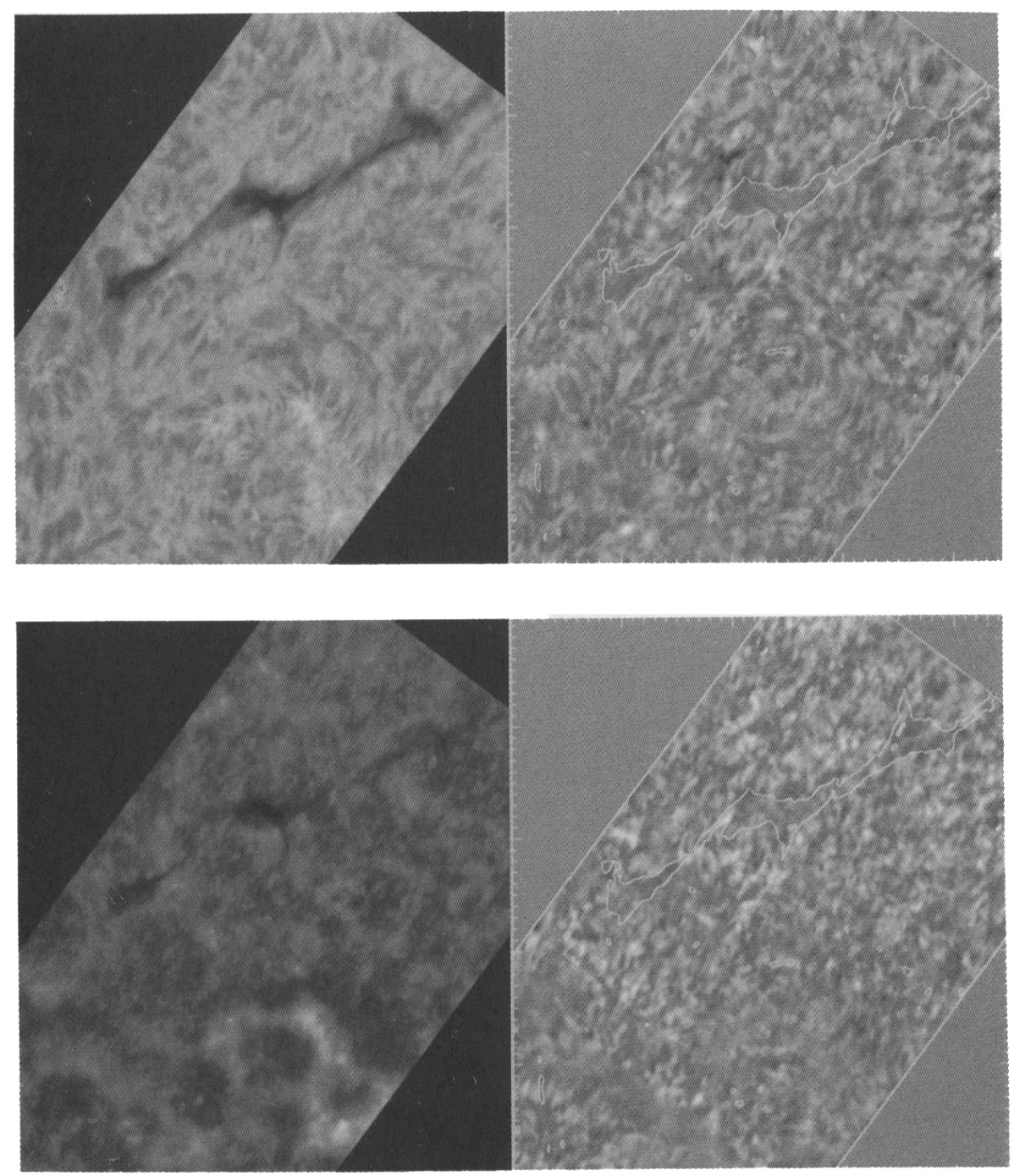

Figure 3. MSDP data obtained at 12:23-12:28 UT (German VTT, Tenerife): top left: $\mathrm{H}_{\alpha}$ intensity; top right: $\mathrm{H}_{\alpha}$ velocities (bright $=$ redshift); bottom left: CaII $8542 \AA$ intensity; bottom right: CaII velocities. The brightness of the network is visible in CaII; in the filament body, velocities are generally upward, especially in $\mathrm{H}_{\alpha}$; close to the "barbs" downward velocities are frequent in CaII (see, for example, the southern foot in the middle part of the filament). 

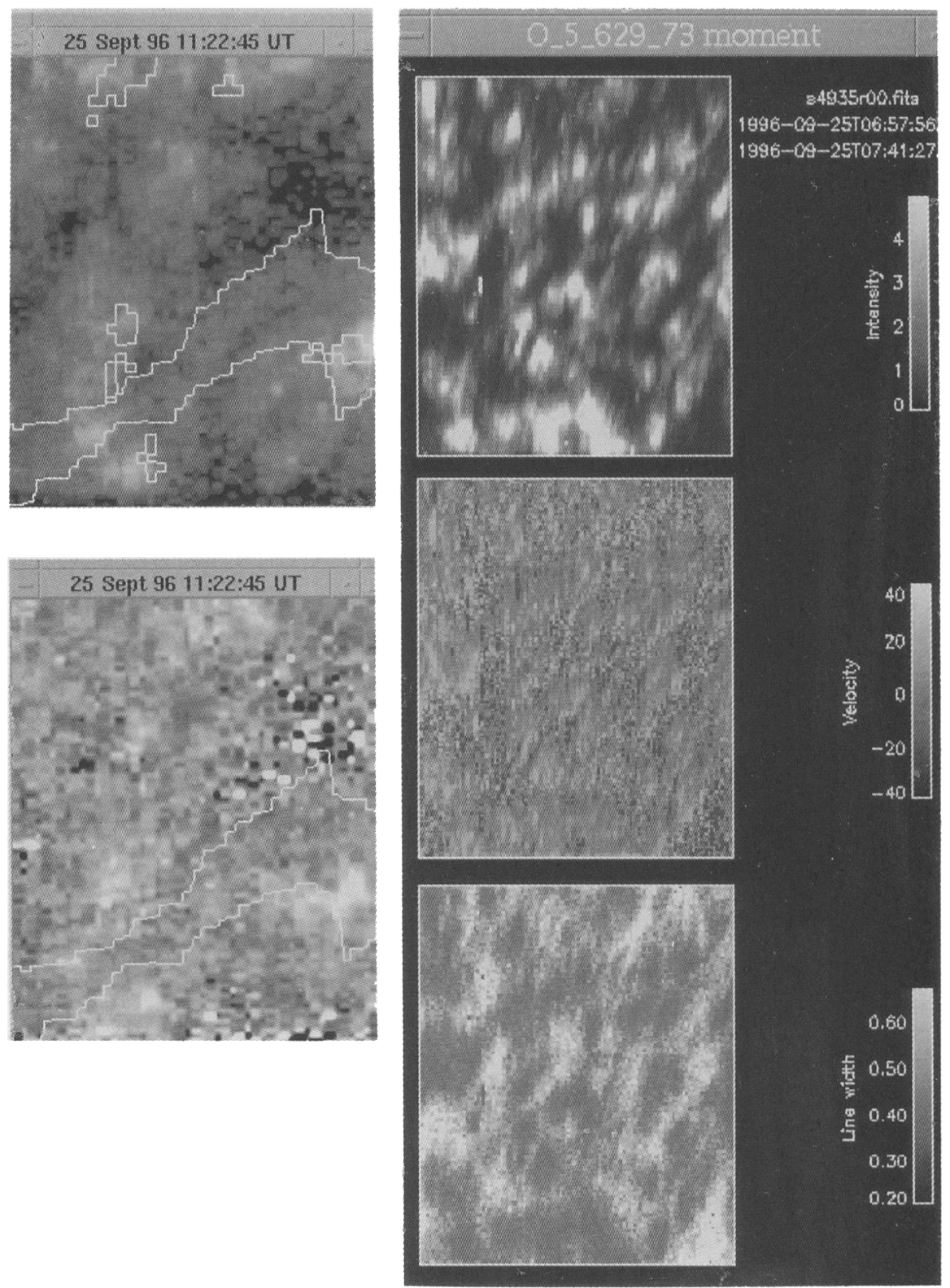

Figure 4. Left: example of SUMER data: Si IV $1394 \AA$ (top: intensities, bottom: velocities, bright $=$ redshift); Right: example of CDS data: OV $629 \AA$ (the scale is smaller than in the SUMER data; the central part includes the full field of Figure 2; bottom; the bright regions correspond to the network visible in CaII (Figure 3)). 